\title{
Anti-Cyclic Citrullinated Peptide Antibody versus HAQ/MDHAQ: Comparing Apples and Oranges?
}

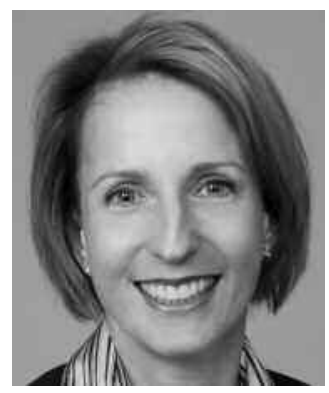

Pincus, et al in the this issue of The Journal address the contentious issue of where anti-cyclic citrullinated peptide antibody (anti-CCP) testing belongs, if at all, in routine clinical practice $^{1}$. The extension of this line of thought is whether or not this test is cost-effective or of real clinical utility to the practicing rheumatologist. The authors address the question whether testing for anti-CCP will improve patient outcomes or be of value in making appropriate clinical therapeutic decisions at the bedside above and beyond the evaluation of function in a systematic fashion. They raise the question whether a composite patient-related non-biologically-based score such as the Health Assessment Questionnaire (HAQ)/Multidimensional (MD) HAQ should trump a laboratory test when evaluating a patient with rheumatoid arthritis (RA). While arguing for the use of this test, the authors note that "Anti-CCP provides a significantly higher hazard ratio than rheumatoid factor (RF) to identify people with early arthritis who will develop progressive disease"2,3, based on greater pooled specificity of $95 \%$ in a metaanalysis of 37 studies, compared to $85 \%$ in 50 studies of RF for $\mathrm{RA}^{3}$. They also argue that the presence of these antibodies may favor the use of treatments that are proven to be highly effective in subsets of patients who are positive for anti-citrullinated peptide antibodies (ACPA), which include anti-CCP ${ }^{4}$.

Perhaps we should not compare apples with oranges, as anti-CCP is a good biomarker predicting sustained inflammatory arthritis and increased risk of erosions in early disease, whereas the HAQ (or other modified versions of the HAQ) is important for evaluating function and can be done repeatedly by the patient. The HAQ, however, does not necessarily perform well in early disease, given that functional impairment early on may be fully reversible ${ }^{5}$ and is not specific for RA, as comorbidities such as degenerative arthritis and advanced age can affect the HAQ score.

In established RA, there is no need to perform anti-CCP if there is preexisting damage, as the largest risk of future RA damage is current radiographic damage ${ }^{6}$. The anti-CCP can help to answer questions such as: (1) Will early inflammatory arthritis be sustained; (2) Is a patient with early disease more apt to acquire damage; and (3) Can sustained remission continue if disease modifying antirheumatic drug (DMARD) therapy is withdrawn? The HAQ addresses the function of the patient at any point in time, and a change in HAQ can help predict worse outcomes, but reflects a mix of RA activity, damage, and other causes of functional impairment.

Along this line of reasoning, whether it be a composite score or the result of a biologically-based assay, a clinical test should enable a clinician to make a diagnosis with more confidence, and choose a therapy that will improve a patient's outcome. The score or result would need to be clinically meaningful at the patient level rather then have significance at a group level. It is probable that this requirement can be fulfilled by both anti-CCP and the HAQ.

However, Pincus, et al question the utility of performing anti-CCP testing outside the realm of the research setting, suggesting that a patient presenting with RA should first receive a trial of methotrexate (MTX) with or without low-dose prednisone, with further investigations being reserved for those who fail to respond. The PROMPT trial nicely demonstrates that fewer erosions occur in MTX-treated patients compared to placebo, but the majority of the benefit on radiographic erosions was in the anti-CCP-positive patients ${ }^{4}$. This would argue for testing first, especially in early undifferentiated arthritis.

The authors imply that it may be more cost-effective to use a clinical index of function (the HAQ/MDHAQ) or composite score encompassing patient-reported outcomes (the MD RAPID III) at the bedside. To substantiate this view the authors note that cheaper tests are more predictive of mortality, referring to data suggesting that the HAQ/MDHAQ are better at predicting mortality and more relevant because HAQ instruments are part of measures of change/response in RA. It should also be noted that others have published data suggesting there is also an association

See Why is anti-CCP included in most routine care while HAQ remains a research tool, page 1563 
of increased mortality in patients who are positive for an anti-CCP test ${ }^{7}$.

In fact the time to order the anti-CCP for maximum utility is when one is uncertain as to diagnosis in early inflammatory arthritis and for early prognosis; but the HAQ can be used at any time even though the routine use of anti-CCP (ordered once in early disease) still needs to be justified in routine clinical practice, given that consensus for the ideal cutoff values of the test has yet to be achieved ${ }^{8}$. As various ACPA assays become available, it remains to be determined which of these will perform optimally in the clinical setting.

In addition, there are several forces and sources of data that have swayed practicing clinicians and even payers to bring anti-CCP testing into prime-time use. In many circumstances payers require objective criteria, such as the presence of joint damage, or positive serological tests, such as anti-CCP or RF, to justify payment for costly therapies. This puts the onus on the practicing clinician to order an anti-CCP test in patients with early inflammatory arthritis not responding to DMARD who have signs of active joint inflammation on clinical examination but negative RF and as yet no evidence of radiographic joint damage. Payers seem less willing to accept a "soft" patient-reported outcome as evidence of RA and are more likely to accept a biomedical "objective" marker of disease to validate the existence of RA. The HAQ is nonspecific but still provides information about the clinical status of the patient. Educating payers about the added value of the HAQ (as being easy to perform, inexpensive, and reliable in tracking patient function over time as well as reflecting response to treatment) may be a real advantage.

A negative anti-CCP identifies a less well-defined group of RA patients in whom one may choose certain therapies over others. It also indicates a higher probability of developing RA in patients who have a positive anti-CCP and yet do not meet criteria for $\mathrm{RA}^{4}$. The American College of Rheumatology criteria do not function well in early disease ${ }^{9}$.

It is tempting given the strength of the evidence to date to resort to an objective test that has been associated with the ability to predict radiographic damage. Clinical studies show that positive anti-CCP is associated more frequently with radiographic progression. In the BeST study, radiographic progression scores were much higher in patients treated with sequential monotherapy ${ }^{10}$, and ACPA positivity better predicted progressive disease in this monotherapy treatment arm versus RF positivity (odds ratio 12.6, 95\% confidence interval 3.0-51.9 for ACPA, compared to OR $4.7,95 \%$ CI $1.5-14.5$ for RF).

Additional reports have confirmed that anti-CCP antibodies at baseline are good predictors of radiographic progression in prospective studies of early RA cohorts with followup periods of 10 years. In a recent study of 238 patients with RA (Norfolk Arthritis Register, 10-year followup),
anti-CCP was the strongest independent predictor of radiographic progression. The presence of anti-CCP antibodies at baseline was a more powerful predictor of both prevalent erosions (OR 2.5) and developing erosions by 5 years (OR 10.2) than was the presence of RF (OR 1.6 and 3.4, respectively) ${ }^{11}$. The presence of RF did not yield significantly higher odds of erosions than anti-CCP alone. However, it was noted that in people with early inflammatory arthritis followed in this Norfolk Arthritis Register, a significant number of anti-CCP-negative patients developed erosions at 5 years (27\% of the prospective cohort), whereas $21 \%$ of patients positive for presence of anti-CCP antibodies had not developed erosions at 5 years ${ }^{11}$. Further, the strength of the association between anti-CCP positivity and development of erosions was damped by the treatment effects of using DMARD and corticosteroids. Finally, results from a recent metaanalysis indicated that positive anti-CCP antibodies in RA were associated with greater radiographic progression, and the risk of radiographic progression was higher for patients with anti-CCP antibody positivity (likelihood ratio 12.5) than for those with positive IgM RF tests (likelihood ratio 4.9$)^{3}$.

Another important decision that frequently arises in routine clinical care: whether or not to withdraw therapy in a patient with low disease activity. Patients frequently seek guidance from their treating physician whether this is an appropriate decision. Patient-reported outcome or composite score cannot predict if treatment can be stopped. However, evidence does show that patients who have a positive anti-CCP will be less likely to withdraw therapy. Van der Woude, et $a l^{12}$ showed that patients who flared after withdrawing therapy were 11 times more likely to be anti-CCP-positive. This has not been shown with the HAQ or MDHAQ.

While it cannot be disputed that utilization of anti-CCP in the research setting will teach us more about how to best use anti-CCP in guidance for management of individual patients, is this not true of any tool currently used in clinical medicine?! Does that mean that clinicians should not employ a test that may help them better identify which patients with inflammatory arthritis are more likely to go on to develop persistent and or destructive arthritis and thus support the utilization of more optimal treatment strategies from the outset? Would a test like anti-CCP not be of particular value in patients who tolerate MTX poorly, especially where there is a high or rising tender and swollen joint count? Up to one-quarter of patients do not adhere to optimal doses of MTX ${ }^{13}$ and most rheumatologists do not perform full joint examinations at the bedside ${ }^{14}$, thus it would stand to reason to encourage use of a biological test to better identify those who should be treated more aggressively, regardless of whether these same patients report poor levels of function in relation to their inflammatory arthritis. It is likely that no single measure gives enough information, and

Personal non-commercial use only. The Journal of Rheumatology Copyright (C) 2009. All rights reserved. 
thus routine measures with patient function, joint counts, and inflammation markers have been advocated ${ }^{15}$.

Do patient-driven outcomes really provide the same or even sufficient information to determine a treatment decision? What about those with RA and concomitant fibromyalgia/pain amplification syndromes? Does the MDHAQ Rapid III predict equally well those who will have persistent or erosive disease? Should both tools not be tested in cohorts to determine their performance in this regard in a research setting? At this time we cannot replace composite scores of function for a serological test unless the 2 correlate well. Such studies have also not yet been done.

The best of both worlds is to do the anti-CCP once in early or suspected RA and follow the patient with validated measures on a regular basis, including the HAQ.

VIVIAN BYKERK, MD, FRCPC, Mount Sinai Hospital, Room 2-005, Box 5, 60 Murray Street,

Toronto, Ontario M5T 3L9, Canada

Address correspondence to Dr.Bykerk; E-mail:vbykerk@mtsinai.on.ca

\section{REFERENCES}

1. Pincus T, Yazici Y, Bergman MJ. Quantitative clinical rheumatology: Why is a test for anti-CCP antibodies included in most routine care for rheumatoid arthritis while a HAQ/MDHAQ remains largely a research tool? J Rheumatol 2009;36:1563-4.

2. van Gaalen FA, Linn-Rasker SP, van Venrooij WJ, et al. Autoantibodies to cyclic citrullinated peptides predict progression to rheumatoid arthritis in patients with undifferentiated arthritis: a prospective cohort study. Arthritis Rheum 2004;50:709-15.

3. Nishimura K, Sugiyama D, Kogata Y, et al. Meta-analysis: diagnostic accuracy of anti-cyclic citrullinated peptide antibody and rheumatoid factor for rheumatoid arthritis. Ann Intern Med 2007;146:797-808

4. Van Dongen H, van Aken J, Lard LR, et al. Efficacy of methotrexate treatment in patients with probable rheumatoid arthritis. A double-blind, randomized, placebo controlled trial. Arthritis Rheum 2007;56:1424-32.

5. Aletaha D, Smolen J, Ward MM. Measuring function in rheumatoid arthritis: identifying reversible and irreversible components. Arthritis Rheum 2006;54:2784-92.
6. Smolen JS, van der Heijde DMFM, St. Clair EW, et al. Predictors of joint damage in patients with early rheumatoid arthritis treated with high-dose methotrexate with or without concomitant infliximab. Results from the ASPIRE trial. Arthritis Rheum 2006;54:702-10.

7. Sihvonen S, Korpela M, Mustila A, et al. The predictive value of rheumatoid factor isotypes, anti-cyclic citrullinated peptide antibodies, and antineutrophil cytoplasmic antibodies for mortality in patients with rheumatoid arthritis. J Rheumatol 2005;32:2089-94.

8. Bossuyt X, Coenen D, Fieuws S, Verschueren P, Westhovens R, Blanckaert N. Likelihood ratios as a function of antibody concentration for anti-cyclic citrullinated peptide antibodies and rheumatoid factor. Ann Rheum Dis 2009;68:287-9.

9. Liao KP, Batra KL, Chibnik L, Schur PH, Costenbader KH. Anti-cyclic citrullinated peptide revised criteria for the classification of rheumatoid arthritis. Ann Rheum Dis 2008;67:1557-61.

10. De Vries-Bouwstra JK, Goekoop-Ruiterman YP, Verpoort KN, et al. Progression of joint damage in early rheumatoid arthritis: association with HLA-DRB1, rheumatoid factor, and anti-citrullinated protein antibodies in relation to different treatment strategies. Arthritis Rheum 2008;58:1293-8.

11. Bukhari M, Thomson $\mathrm{W}$, Naseem H, et al. The performance of anti-cyclic citrullinated peptide antibodies in predicting the severity of radiologic damage in inflammatory polyarthritis: Results from the Norfolk Arthritis Register. Arthritis Rheum 2007;56:2929-35.

12. van der Woude D, Young A, Brand R, et al. Prevalence and predictive factors for DMARD-free remission in RA. Lessons from 2 large early arthritis cohorts [abstract]. Arthritis Rheum 2008;58 Suppl:S760.

13. Fransen J, Lann RFJM, van der Laar MAFJ, et al. Influence of guideline adherence on outcome in a randomised controlled trial on the efficacy of methotrexate with folate supplementation in rheumatoid arthritis. Ann Rheum Dis 2004;63;1222-6.

14. Pincus T. Limitations of a quantitative swollen and tender joint count to assess and monitor patients with rheumatoid arthritis. Bull NYU Hosp Jt Dis 2008;66:216-23.

15. Combe B, Landewe R, Lukas C, et al. EULAR recommendations for the management of early arthritis: report of a task force of the European Standing Committee for International Clinical Studies Including Therapeutics (ESCISIT). Ann Rheum Dis 2007;66:34-45.

J Rheumatol 2009;36:1565-7; doi:10.3899/jrheum.090673 